\title{
Childhood maltreatment and emotional distress: The role of beliefs about emotion and psychological inflexibility
}

\author{
Aslı Burçak Taşören ${ }^{1}$ \\ Accepted: 1 December 2021 \\ (c) The Author(s), under exclusive licence to Springer Science+Business Media, LLC, part of Springer Nature 2021
}

\begin{abstract}
The current study aimed to investigate the mediating role of negative beliefs about emotion and psychological inflexibility on the relationship between childhood maltreatment and emotional distress. A total of 519 participants completed the Childhood Trauma Questionnaire (CTQ), Depression Anxiety Stress Scale-21 (DASS-21), the Leahy Emotional Schema Scale II (LESS-II), and the Acceptance and Action Questionnaire-II (AAQ-II). All scales were significantly correlated. Two mediation analyses were tested. In the first model negative beliefs about emotion and psychological inflexibility mediated the relationship between childhood maltreatment and emotional distress measured by DASS-21 total score. In the second model, negative beliefs about emotion and psychological inflexibility mediated the relationship between childhood maltreatment and depression, anxiety, and stress measured by the subscales of DASS-21. Results suggest that maltreatment in childhood is associated with the individuals' approaches, plans and strategies in response to emotions, and psychological inflexibility which together further determine emotional distress.
\end{abstract}

Keywords Childhood maltreatment $\cdot$ Emotional distress $\cdot$ Emotional schemas $\cdot$ Psychological inflexibility $\cdot$ Experiential avoidance

\section{Introduction}

The Emotional Schema Model is a social cognitive model focusing on the beliefs about emotions and how they function. These include interpretation, conceptualization, evaluation, and appraisal of emotions, which in turn define how particular emotions are regulated. According to the model, emotions are linked to arrays of many other emotions and any experience of emotion usually comes with an interpretation such as "I have to control my emotion", "Does my anxiety make sense". These are called "theories of emotion" and they form emotional schemas which are the individuals' beliefs about his and others' emotions (Leahy, 2015, 2018, 2019). Understanding these beliefs about emotion helps in understanding and explaining individual emotional tendencies (Veilleux et al., 2021) and emotional problems are closely related to these personal appraisals, evaluations, and beliefs. Relatedly, understanding how emotions function,

Aslı Burçak Taşören

elizasli@icloud.com; abtasoren@medipol.edu.tr

1 Istanbul Medipol Universitesi Guney Kampus, Goztepe Mah. Kavacik, Beykoz, Istanbul, Turkey and helping the individual gain self-awareness concerning beliefs about emotion, is essential in the helping process (Leahy, 2015).

Individuals who employ negative emotional schemas tend to refrain from emotionally laden experiences which is a characteristic closely related to avoidance based coping (Edwards, et al., 2020) and experiential avoidance which is thoroughly addressed in Acceptance and Commitment Therapy (ACT). Experiential avoidance is defined as a process in which thoughts and language lead a person to avoid private experiences, such as emotions, sensations, memories, and thoughts, with the rule that people should get rid of what they don't like. This may be helpful for a while, but in the end it strengthens the presence of the problem which the person is trying to avoid (Hayes \& Smith, 2005). Instead of cognitive or emotional avoidance, Hayes et al. (1996) prefers to use the more broad term "experiential avoidance", since memories, thoughts, and emotions are closely linked in the process.

According to ACT, psychological inflexibility and trying to avoid or eliminate negative feelings is the core of unhappiness and psychopathology, whereas living one's life in accordance with values requires flexibility (Luoma et al., 
2017). Psychological flexibility requires an individual to choose and change a behavior depending on how adaptive, functional and consistent with values it is, and helps balance needs and wishes (Tirch et al. 2012; Kashdan \& Rottenberg, 2010). It is a model designed to understand human functioning and intervene in behavioral change which is at the core of ACT (Hayes et al., 2012).

\section{Childhood Maltreatment, Emotional Schemas, Psychological Flexibility, and Mental Health}

History of childhood maltreatment is known to be related with internalizing and externalizing problems in adolescence (Cohen \& Thakur, 2021; Moraes et al., 2018), as well as mental health problems in adulthood, including anxiety and depression (Goodman et al., 2017; Li et al., 2020a, b, 2021; Sheffler et al., 2019), and poses a risk for abusing one's child (Liu et al., 2019.) Many factors, including being unresponsive to the child's emotional needs, being hostile against or inhibiting the child's emotional expression are negatively related with healthy emotional development (Krause et al., 2003), and may lead to self-blame and internalizing of parents' negative criticism (Coates \& Messman-Moore, 2014; Pearce \& Pezzot-Pearce, 2007).

The relationship between problems in emotion regulation and mental health problems and psychopathology is well documented in the literature (Veilleux et al., 2021), relatedly, accepting and tolerating negative emotions is associated with less psychological symptoms (Berking et al., 2012). Maladaptive coping behaviors which include blaming, attacking, surrendering, withdrawing, and clinging, are learned early in life and may result in interpersonal problems throughout the individuals' life and can also be driven by experiential avoidance (McKay et al., 2012).

Emotion regulation was found to mediate the relationship between emotional schemas and post-traumatic stress symptoms (Mazloom et al., 2016), and negative beliefs about emotion were found to be related to depression (Rezaei et al., 2016) and anxiety (Edwards et al., 2020). Emotional schemas and metacognitive beliefs can act in the maintenance of worry, alone and together with experiential avoidance (Akbari \& Khanipour, 2018), which in turn is found to mediate the relationship between negative emotional schemas and depression (Rezaei et al., 2016). Experiential avoidance was found to be higher among female victims of child sexual abuse, compared to women without a history of abuse (Batten et al., 2001) and mediated the association between history of childhood emotional abuse and partner trust (Baugh et al., 2019). Experiential avoidance can be viewed as a self-regulation strategy which may link various risk factors across mental health problems including worry (Akbari \& Khanipour, 2018; Fischer et al., 2016) and on the other hand psychological flexibility may help in recovering form negative emotions (Predatu et al., 2020).

\section{The Present Study}

The aim of the present study is to understand the role of beliefs about emotions and psychological flexibility in the relationship between childhood maltreatment and negative emotional states. Relationships with parents have various effects on how a child copes with emotions and also shapes beliefs concerning emotions, both of self and others. It is believed that developmental outcomes related with childhood maltreatment lead to negative emotional states, including stress, symptoms of anxiety and depression. Emotional Schema Therapy (EST) emphasizes avoiding and suppressing emotions (Leahy, 2015) and much like the EST, ACT particularly emphasizes the negative consequences of experiential avoidance, which is avoiding certain emotions, feelings, sensations, thoughts, and behaviors, and trying to change the form, rate and contexts in which these private experiences occur. Experiential avoidance is one of the main components of psychological inflexibility (Hayes et al., 1996) and working with experiential avoidance in ACT, is found to be effective with individuals with a history of childhood trauma (Makriyianis et al., 2019). These characteristics are interrelated, formed and shaped early in life and are believed to be associated with emotional distress.

In the present study it was hypothesized that negative beliefs about emotion and psychological inflexibility would mediate the relationship between childhood maltreatment and emotional distress. It is believed that childhood maltreatment is associated with negative conceptualizations, beliefs, thoughts about emotion, and ineffective strategies in coping with emotions and psychological inflexibility, which is further related with negative affect. A mediation model was tested to assess the mediating role of negative beliefs about emotions assessed through emotional schemas, and psychological inflexibility on the relationship between childhood maltreatment and emotional distress in terms of depression, anxiety, and stress symptoms.

\section{Methods}

\section{Participants and Procedure}

A sample of 519 adults (384 female and 135 male) participated in the study. Inclusion criteria included being at least 18 years of age, having consent to participate in the study, and completing all questionnaire items. Participants were asked whether they were diagnosed with any psychiatric disorder, exclusion criteria included being diagnosed with schizophrenia spectrum and other psychotic disorders. 
The mean age was $33.3(\mathrm{SD}=13.4)$ and ranged from 18 to 76 (213 participants aged $18-24 ; 146$ participants aged 25-39; 110 participants aged 40-54; 50 participants aged 55-76). Most of the sample consisted of university graduates $(37.6 \%)$, followed by university students $(27.5 \%)$, highschool graduates $(13.1 \%)$, graduates from masters or $\mathrm{PhD}$ programs $(8.3 \%)$ and finally primary and secondary school graduates $(7.9 \%$ and $5.2 \%) ; 309(59.5 \%)$ were single and 210 $(40.5 \%)$ of the participants were married.

Ethical approval for the study was obtained. Convenience sampling method was employed, the instruments were sent online through a Google form, including the purpose of the study, name and contact information of the researcher and informed consent. The researcher sent the forms to contacts and students which further sent them to acquaintances. Four separate forms were created in which instruments were introduced in differing order (first form LESS-II, DASS21, AAQ-II, CTQ; second form DASS-21, LESS-II, CTQ, AAQ-II; third form LESS-II, CTQ, AAQ-II-II, DASS-21; fourth form AAQ-II, DASS-21, LESS-II, CTQ) in order to eliminate any shortcoming which may arise from the order in which the instruments were given (e.g. such as getting bored after a point). Also one control item (e.g. answer this item as "almost always true") was added in each form in order to detect and eliminate random responding.

\section{Measures}

\section{History of Childhood Maltreatment}

The Childhood Trauma Questionnaire (Bernstein et al., 1994) was used to assess history of childhood maltreatment. The CTQ consists of 28 items and five subscales, namely emotional abuse, physical abuse, sexual abuse, emotional neglect, and physical neglect. Higher scores indicate an increase in abuse and neglect. Cronbach's Alpha coefficients of the inventory range between 0.79 to 0.94 .

Şar et al. (2012) revised the Turkish form and changed certain expressions to fit the Turkish culture. They also expanded the scale by adding an overprotection/overcontrol subscale consisting of 5 items. This dimension measures interference, restrictive and intrusive behavior on the part of the parents, which is common in the Turkish culture. The Cronbach's alpha coefficient for the total scale is 0.87 .

\section{Emotional Distress}

Depression Anxiety Stress Scale-21 (DASS-21) is a 21 item self-report measure developed to assess the severity and frequency of three negative emotional states, namely, depression, anxiety, and stress/tension over the past week (Lovibond \& Lovibond, 1995). DASS-21 is a 4-point rating scale, and each emotional state is measured with 7 items.
The internal consistency measured with the Cronbach's alpha was 0.81 for depression; 0.73 for anxiety, and 0.81 for stress.

The Turkish standardization study was conducted by Yildirim et al. (2018) and the Cronbach's alpha was 0.89 for depression, 0.87 for anxiety, and 0.90 for stress.

\section{Beliefs and Thoughts about Emotions}

The Leahy Emotional Schema Scale II (LESS-II) is a shorter 28 item version of the LESS (Leahy, 2002) which measures the experience, evaluation, and belief about emotions, attributions of emotions, and strategies for emotion regulation and coping, through 14 schemas.

The Turkish form is divided into two categories, namely adaptive emotional schemas and rigid emotional schemas. Adaptive emotional schemas consists of the Validation, Comprehensibility, Higher Values, Control, Consensus, Acceptance of Feelings schemas: rigid emotional schemas, consists of the Guilt, Simplistic View of Emotion, Numbness, Rational, Duration, Rumination, and Blame schemas (Batmaz \& Özdel, 2015). A composite score for total negative beliefs about emotions can be obtained by reversing the scores of the adaptive emotional schemas and adding them with the rigid emotional schemas.

Cronbach's alpha is 0.76 for the Turkish adaptation study (Batmaz \& Özdel, 2015).

\section{Psychological Flexibility}

The Acceptance and Action Questionnaire-II (AAQ-II) is a 7 item, 7 point rating scale designed to measure psychological inflexibility (Bond et al., 2011). The internal consistency measured with the Cronbach's alpha was 0.84 both for the original scale and the Turkish version (Yavuz et al., 2016).

\section{Data Analyses}

Data was screened for outliers, random responding, and missing values. Outliers were detected, and 29 scores were deleted from the data set. Normality tests were performed for total scores of CTQ, LESS-II, AAQ-II, and DASS-21. Batmaz and Özdel (2015) proposed a composite "negative beliefs about emotions" score for the Turkish form, and the AAQ-II is composed of a single dimension, therefore item parceling procedure (balancing approach) was applied for LESS-II and AAQ-II to create observed variables. LESS-II scale was transformed into a 4-factor structure and the AAQII scale into a 2-factor structure.

Descriptive statistics were computed for study variables. In order to conduct a measurement model, Pearson's correlation was computed for total scores to measure relationships between latent variables. First, the measurement model was 
tested, model fit indices confirmed a reflective measurement model, and it was concluded that the latent variables caused the measurement of the indicator variables for the present data set.

Two mediation models were tested through structural equation modeling (SEM) in which the effects of childhood maltreatment and the mediator variables (emotional schemas and psychological inflexibility) on emotional distress were assessed. The sexual abuse subscale of the CTQ wasn't predicted by the total scale and thus was eliminated from the model. The remaining subscales were used as manifest indicators for the childhood maltreatment latent variable in both models. Again, in both models, there were two mediator variables: negative beliefs about emotion was modeled as a latent variable with LESS-II as the indicator; and psychological inflexibility/experiential avoidance was modeled as a latent variable with AAQ-II as the indicator. In the first model, DASS-21 total scale was used as an indicator for emotional distress latent variable, and in the second model depression, anxiety, and stress subscales of DASS-21 were used distinctively as indicators of emotional distress latent variable.

Several goodness-of-fit indices were used to assess the overall fit of the hypothesized model with the actual data: namely $\chi^{2}$, standardized root mean square residual (SRMR), the root mean square error of approximation (RMSEA), the comparative fit index (CFI), and the goodness of fit index (GFI). Cut-off for good fit is 0.08 or lower for RMSEA and
SRMR, and 0.90 or higher for CFI and GFI. This model's fit statistics indicated a good fit for the data. Also, $\chi^{2} /$ degree of freedom which should be between 2 to 5 , was acceptable (Tabachnick \& Fidell, 2001). SPSS 25.0 and LISREL 8.80 were used for data analyses.

\section{Results}

Descriptive statistics are found in Table 1.

Correlations between latent variables are listed in Table 2. Childhood maltreatment measured with CTQ, emotional schemas measured with LESS-II, psychological inflexibility/experiential avoidance measured with AAQ-II, emotional distress measured with DASS-21 total score, and depression, anxiety and stress measured distinctively by the subscales were positively and significantly correlated with each other.

The CTQ consists of 6 subscales, the sexual abuse subscale wasn't predicted by the total scale and thus was eliminated from the model and the analysis was conducted with the remaining 5 subscales. As a result of the repeated analysis, it was determined that the $t$ values of the factors were significant and between 7.72 to 20.25 , and the error variance values were below 0.95 (between 0.31 to 0.81 ). As a result of the analysis, when the fit indices of the scale were examined, it was found that the $\mathrm{S}-\mathrm{B} \chi 2$ value was significant, $\mathrm{S}-\mathrm{B} \chi^{2}$ $(4)=17.89$, RMSEA $=0.08, p=0.001$. When the other fit indices were examined, it was found that the $S-B \chi 2 / \mathrm{sd}$
Table 1 Range, mean, standard deviation, skewness, kurtosis of study variables

Table 2 Correlations between CTQ, LESS-II, AAQ-II, DASS21 total scale and DASS-21 subscales

\begin{tabular}{|c|c|c|c|c|c|c|c|c|}
\hline \multirow[t]{2}{*}{ Variables } & \multirow[t]{2}{*}{$\mathrm{n}$} & \multirow[t]{2}{*}{ Min-Max } & \multirow[t]{2}{*}{ M } & \multirow[t]{2}{*}{ SD } & \multicolumn{2}{|l|}{ Skewness } & \multicolumn{2}{|l|}{ Kurtosis } \\
\hline & & & & & Statistics & SE & Statistics & SE \\
\hline CTQ & 519 & $30-77$ & 46.75 & 11.15 & 0.68 & 0.11 & -0.32 & 0.21 \\
\hline LESS & 519 & $45-127$ & 84.49 & 15.50 & 0.06 & 0.11 & -0.34 & 0.21 \\
\hline AAQ & 519 & $7-42$ & 19.46 & 7.69 & 0.37 & 0.11 & -0.67 & 0.21 \\
\hline DASS & 519 & $0-43$ & 17.90 & 9.19 & 0.46 & 0.11 & -0.16 & 0.21 \\
\hline Depression & 519 & $0-19$ & 6.23 & 3.64 & 0.69 & 0.11 & 0.17 & 0.21 \\
\hline Anxiety & 519 & $0-14$ & 4.44 & 3.23 & 0.71 & 0.11 & -0.02 & 0.21 \\
\hline Stress & 519 & $0-20$ & 7.24 & 3.74 & 0.45 & 0.11 & 0.18 & 0.21 \\
\hline
\end{tabular}

\begin{tabular}{lccccccc}
\hline & $\mathbf{1}$ & $\mathbf{2}$ & $\mathbf{3}$ & $\mathbf{4}$ & $\mathbf{5}$ & $\mathbf{6}$ & $\mathbf{7}$ \\
\hline 1.CTQ & - & & & & & & \\
1.LESS-II & $0.34^{*}$ & - & & & & \\
1. AAQ-II & $0.40^{*}$ & $0.61^{*}$ & - & & & \\
1.DASS-21 & $0.28^{*}$ & $0.55^{*}$ & $0.65^{*}$ & - & & \\
1.Depression & $0.26^{*}$ & $0.55^{*}$ & $0.59^{*}$ & $0.86^{*}$ & - & \\
1.Anxiety & $0.22^{*}$ & $0.39^{*}$ & $0.52^{*}$ & $0.83^{*}$ & $0.53^{*}$ & - & \\
1.Stress & $0.24^{*}$ & $0.47^{*}$ & $0.58^{*}$ & $0.91^{*}$ & $0.68^{*}$ & $0.66^{*}$ & - \\
\hline
\end{tabular}

${ }^{*} p<0.01, C T Q$ The Childhood Trauma Questionnaire, LESS-II Leahy Emotional Schemas Scale II, AAQ-II Acceptance and Action Questionnaire-II, DASS-21 Depression, Anxiety and Stress Scale-21 
ratio was $4.47, \mathrm{SRMR}=0.04, \mathrm{CFI}=0.98$, and $\mathrm{GFI}=0.97$, meaning that the measurement model was verified considering the fit indices.

The $t$ values of the 4 factors the LESS-II were significant and between 13.01 to16.98, and the error variance values were below 0.95 (between 0.46 to 0.65 ). As a result of the analysis, when the fit indices of the scale were examined, it was found that the $\chi^{2}$ value was significant, $\chi^{2}(2)=8.00$, $\mathrm{RMSEA}=0.076, \mathrm{p}=0.02$. Fit indices are as follows: the $\chi 2$ / $\mathrm{sd}$ ratio was $4, \mathrm{SRMR}=0.02, \mathrm{CFI}=0.99$ and $\mathrm{GFI}=0.99$. Thus, the measurement model was verified considering the fit indices.

The $t$ values of the 2 factors of AAQ-II were significant and between 56.88 to 78.85 , and the error variance values were below 0.95 (between 0.09 to 0.16 ). The fit indices of the scale showed that the measurement model was saturated, $\mathrm{S}-\mathrm{B} \chi 2(0)=0.00, \mathrm{RMSEA}=0.00, \mathrm{p}=1$. The DASS-21 scale consists of 3 factors, the measurement model was saturated, $\mathrm{S}-\mathrm{B} \chi 2(0)=0.00, \mathrm{RMSEA}=0.00, \mathrm{p}=1$.

Structural equation modeling was used to test the mediating role of emotional schemas and psychological inflexibility

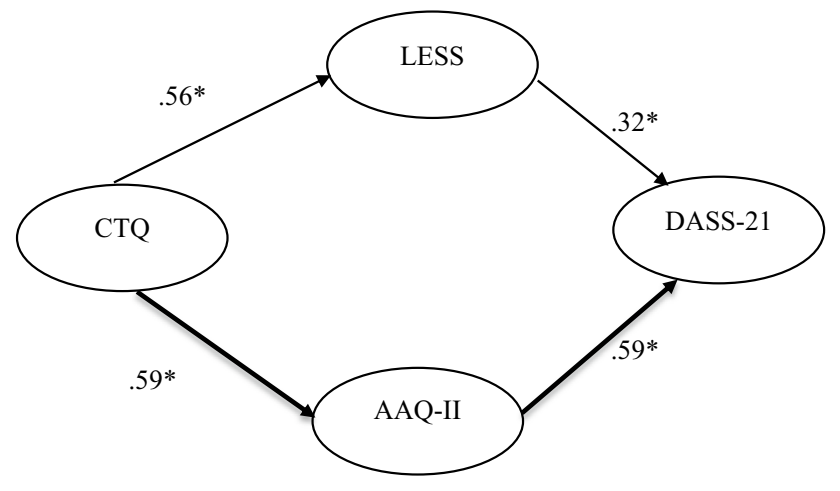

Fig. 1 Structural equation model analysis testing the effect of childhood maltreatment on emotional distress through the mediating role of emotional schemas and psychological inflexibility. Note. All standardized coefficients are significant $(* p<.05)$ on the relationship between childhood maltreatment and emotional distress.

Fit indices suggest that the model fit the data well $\left(\chi^{2} /\right.$ $\mathrm{df}=4.42 p=0$ 0.00, RMSEA $=0.081, \mathrm{SRMR}=0.011$, $\mathrm{CFI}=0.95, \mathrm{GFI}=0.89$ ). Figure 1 shows standardized coefficients for the measurement and structural model.

There were two indirect paths between childhood maltreatment and emotional distress, namely through emotional schemas and psychological inflexibility. All paths except the direct path from childhood maltreatment to emotional distress were significant at $\mathrm{p}<0.05$. Standardized coefficients illustrating latent variable relationships for each model were significant: the path from childhood maltreatment to emotional schemas $(\beta=0.56, p<0.05)$ and from emotional schemas $(\beta=0.32, \mathrm{p}<0.05)$ to emotional distress; the path from childhood maltreatment to psychological inflexibility $(\beta=0.59, \mathrm{p}<0.05)$, and from psychological inflexibility $(\beta=0.59, \mathrm{p}<0.05)$ to emotional distress. The direct path from childhood maltreatment $(B=-0.11, \mathrm{p}>0.05)$ to emotional distress was not significant, suggesting that emotional schemas and psychological inflexibility fully mediated the relationship between childhood maltreatment and emotional distress.

Also mediation effect size in the structural models were assessed. Effect sizes correspond to those defined by Cohen as small, medium, and large $(0.00=$ null effect, $0.14=$ small effect, $0.36=$ medium effect, $0.59=$ large effect; Cohen, 1988, see Fig. 1 and Table 3). CTQ has a direct effect on LESS-II $(\beta 1=0.56, p<0.05)$ and AAQ-II $(\beta 1=0.59$, $\mathrm{p}<0.05)$, and LESS-II and AAQ-II have a direct effect on DASS-21 $(\beta 2=0.32, p<0.05 ; \beta 2=0.32, p<0.05)$. The direct effect of CTQ on DASS-21 $(\beta 4=0.07, \mathrm{p}<0.05)$ became non-significant when LESS-II $(\beta 3=-0.11, \mathrm{p}>0.05)$ was added as mediator. Also the direct effect of CTQ on DASS-21 $(\beta 4=0.24, \mathrm{p}<0.05)$ became non-significant when AAQ-II $(\beta 3=-0.11, \mathrm{p}>0.05)$ was added as mediator. In this case it can be said that LESS-II and AAQ-II fully mediate the relationship between CTQ and DASS-21.

Table 3 Assessment of mediation effect in the structural models

\begin{tabular}{lllllllll}
\hline & $\beta_{1}$ & $\beta_{2}$ & $\beta_{3}$ & Effect size & Effect $\%$ & $\mathrm{t}$ & $\mathrm{p}$ & Decision \\
\hline $\mathrm{CTQ} \rightarrow$ LESS-II $\rightarrow$ DASS-21 & 0.56 & 0.32 & -0.11 & 0.07 & -1.63 & -1.11 & 0.058 & Small effect size, full mediation \\
$\mathrm{CTQ} \rightarrow$ AAQ-II $\rightarrow$ DASS-21 & 0.59 & 0.32 & -0.11 & 0.24 & -3.16 & -1.11 & 0.061 & Medium effect size, full mediation \\
$\mathrm{CTQ} \rightarrow$ LESS-II $\rightarrow$ Depression & 0.57 & 0.44 & -0.12 & 0.13 & -2.09 & -1.21 & 0.075 & Small effect size, full mediation \\
$\mathrm{CTQ} \rightarrow$ AAQ-II $\rightarrow$ Depression & 0.61 & 0.47 & -0.12 & 0.17 & -2.39 & -1.21 & 0.069 & Medium effect size, full mediation \\
$\mathrm{CTQ} \rightarrow$ LESS-II $\rightarrow$ Anxiety & 0.57 & 0.12 & -0.11 & -0.04 & -0.62 & -1.06 & 0.094 & Small effect size, full mediation \\
$\mathrm{CTQ} \rightarrow$ AAQ-II $\rightarrow$ Anxiety & 0.59 & 0.58 & -0.11 & 0.23 & -3.11 & -1.06 & 0.110 & Medium effect size, full mediation \\
$\mathrm{CTQ} \rightarrow$ LESS-II $\rightarrow$ Stress & 0.57 & 0.21 & -0.11 & 0.01 & -1.09 & -1.12 & 0.074 & Small effect size, full mediation \\
$\mathrm{CTQ} \rightarrow$ AAQ $-\mathrm{II} \rightarrow$ Stress & 0.61 & 0.59 & -0.11 & 0.25 & -3.27 & -1.12 & 0.077 & Medium effect size, full mediation \\
\hline
\end{tabular}

Note. $\beta_{1}$ and $\beta_{2}$ are standardized coefficients of direct effect, $\beta_{3}$ is standardized coefficients of indirect effect 
The second model was tested with the subscales of DASS-21. Standardized coefficients illustrating latent variable relationships for each model were significant: the path from childhood maltreatment to emotional schemas $(B=0.57, \beta=0.57, \beta=0.57, p<0.05)$ and from emotional schemas to depression $(\beta=0.44, p<0.05)$, anxiety $(\beta=0.12, p<0.05)$, and stress $(\beta=0.21, p<0.05)$; the path from childhood maltreatment to psychological inflexibility $(\beta=0.61, \beta=0.59, \beta=0.61, p<0.05)$, and from psychological inflexibility to depression $(\beta=0.47, p<0.05)$, anxiety $(\beta=0.58, p<0.05)$, and stress $(\beta=0.59, p<0.05)$. In the structural model, the direct path from childhood maltreatment to depression, anxiety and stress was investigated in order to examine the mediation effect. As a result, emotional schemas and psychological inflexibility were found to fully mediate the relationship between childhood maltreatment and depression, anxiety, and stress (see Fig. 2). As can be seen in Table 3, mediation effect size is the same in both models, confirming same effect size in the model with the DASS-21 total score and DASS-21 subscales. The direct effect of CTQ on DASS-21 depression, anxiety, and stress subscales $(\beta 4=0.13, \mathrm{p}<0.05$; $\beta 4=-0.04, p<0.05 ; \beta 4=0.01, p<0.05)$ became non-significant when LESS-II $(\beta 3=-0.12, p>0.05 ; \beta 3=-0.11$, $\mathrm{p}>0.05 ; \beta 3=-0.11, \mathrm{p}>0.05)$ was added as mediator. Also the direct effect of CTQ on DASS-21 depression, anxiety, stress subscales $(\beta 4=0.17, \mathrm{p}<0.05 ; \beta 4=0.23, \mathrm{p}<0.05$; $\beta 4=0.25, \mathrm{p}<0.05)$ became non-significant when AAQII $(\beta 3=-0.12, p>0.05 ; \beta 3=-0.11, p>0.05 ; \beta 3=-0.11$, $\mathrm{p}>0.05$ ) was added as mediator. In this case it can be said that LESS-II and AAQ-II fully mediate the relationship between CTQ and DASS-21 subscales.

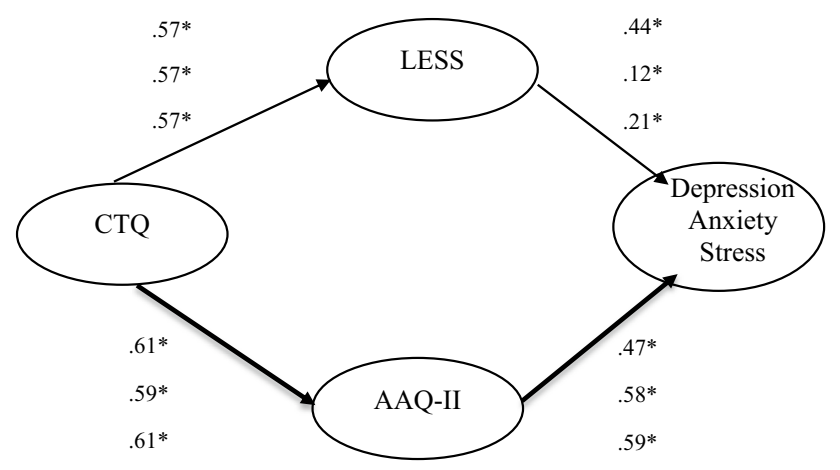

Fig. 2 Structural equation model analysis testing the effect of childhood maltreatment on depression, anxiety, and stress through the mediating role of emotional schemas and psychological inflexibility. Note. All standardized coefficients are significant $(* p<.05)$

\section{Discussion}

In the current study, two potential underlying mechanisms, namely negative beliefs about emotion and psychological inflexibility, through which childhood maltreatment is thought to contribute to emotional distress, were assessed. For this purpose, a mediation model was tested to examine the mediating role of emotional schemas and psychological inflexibility on the relationship between childhood maltreatment and emotional distress. Initial correlational analyses revealed associations between all variables and especially a strong association between emotional distress and psychological inflexibility. The tested model showed the mediating effect of emotional schemas and psychological inflexibility on the relationship between childhood maltreatment and emotional distress. Increased childhood maltreatment was associated with increased negative beliefs about emotion and psychological inflexibility, which in turn determined emotional distress.

A second model tested whether the relationship between childhood maltreatment, and depression, anxiety and stress symptoms distinctly, were mediated by emotional schemas and psychological inflexibility. This second model was tested to see whether the model was justified when subscales of emotional distress were tested separately. Results showed that the model was justified with the subscales and that emotional schemas and psychological inflexibility fully mediated the relationship between childhood maltreatment and emotional distress, regardless of type of distress. Thus, results show that depression, anxiety and stress symptoms were all determined by the stated associations, suggesting that all three types of symptoms were determined by the association between childhood maltreatment, negative beliefs about emotion and psychological inflexibility.

Childhood maltreatment brings about many upsetting, traumatic experiences, which are well documented risks for emotional distress and later mental health problems (Edalati et al., 2019; Li et al., 2020a, b; Spinhoven et al., 2016), and relatedly problems with regulation of emotions and psychological inflexibility (Caldwell et al., 2014; Reffi et al., 2019; Coates \& Messman-Moore, 2014; Boykin et al., 2018). Given the broadness of the adverse effects related with maltreatment, it is essential to determine the mechanisms by which these negative outcomes reflect on negative affect. From the tested models, it can be assumed that negative experiences related with maltreatment may contribute to the many factors which shape the way the child experiences emotions and forms conceptualizations in face of negative emotions. Given that problematic relations with parents are due to create upsetting emotions, the child may appraise emotions as experiences which should be avoided, due to the nature of emotions being experienced, or even take them as 
something that is bad and harmful. It can be said that in this process, the child will form conceptualizations and beliefs related to emotions which Leahy (2002) calls emotional schemas, and will try to find ways to deal with these experiences. Given that childhood maltreatment is not an isolated experience and that negative and dysfunctional relationships with parents or caregivers will affect the child in all developmental areas, the ongoing problems in the relationship with them will have a hold of the child in many ways. With time the growing child may repeatedly experience negative emotions related to relationships with parents and may also be vulnerable in face of other social relationships and can even try to find ways to escape socialization. These negative beliefs about emotion are interrelated with emotion processing, they interact with emotion regulation, and may lead to avoidant coping (Edwards et al., 2020) which is also related with experiential avoidance (Karekla \& Panayiotou, 2011). In turn, these emotional schemas may interact with experiential avoidance to increase negative affect in face of distressing events. These processes together will shape emotional functioning and as stated, the interrelationships will lead to various outcomes including emotional distress.

As expected, the present findings reveal an association between negative beliefs about emotions and symptoms of depression, anxiety, and stress as well as increased history of childhood maltreatment. In the present study the short form of the Leahy emotional schema scale was used in which each emotional schema is assessed with only two items, which limits studying the 14 dimensions of emotional schemas distinctively. Studies in which emotional schemas were studied distinctively, results show that most of the emotional schemas are related with emotional distress including depression (eg. less validation, higher values, control, consensus with the emotions of others, higher simplistic view of emotions, numbness, rationality, rumination, blame, guilt, duration) and anxiety (eg. rumination, guilt, simplistic view of emotions, invalidation, blaming, duration, lack of control, less consensus of emotions and acceptance) suggesting that maladaptive emotional schemas are common in depression and anxiety (Batmaz et al., 2014; Leahy, 2002; Tirch et al., 2012). In addition to negative emotional states, certain maladaptive beliefs related to emotions, such as believing that emotions last forever, judging emotions as bad and that they are difficult to understand, may make people feel that they can't handle their emotions, and may result in higher levels of emotion dysregulation and symptoms of psychopathology (Veilleux et al., 2020; Veilleux et al., 2021.

The findings of the present study also revealed an association between psychological inflexibility and symptoms of depression, anxiety, and stress as well as increased history of childhood maltreatment. These results extend previous research findings linking psychological inflexibility and emotional distress, including depression and anxiety
(Fonseca et al., 2020; Biglan et al., 2015; Bardeen et al., 2014; Tull \& Gratz, 2008; Moroz \& Dunkley, 2019; Richardson \& Jost, 2019).

There are fewer studies investigating the link between psychological inflexibility and its association with childhood traumas. The present study is in line with several studies in which psychological inflexibility was found to be related with childhood abuse (Batten et al., 2001), and mediated the association between history of childhood maltreatment and negative emotional state (Reddy et al., 2006; Richardson \& Jost, 2019), depressive symptom severity in depression (Ghazanfari et al., 2018), behavioral problems (Roche et al., 2019), PTSD (Ramaghani et al., 2019), and trauma related psychological distress (Rosenthal et al., 2005). On the other hand, one study failed to find a relationship between adverse childhood experiences and experiential avoidance (Makriyianis et al., 2019), while another study reported increased psychological flexibility in individuals with multiple early traumas, suggesting that early trauma helped in developing resilience (Richardson \& Jost, 2019).

In terms of the relationship between emotional schemas and psychological inflexibility, the results of the present study indicate an association between negative beliefs about emotion and psychological inflexibility, and that they act together in determining emotional distress. Similarly, Silberstein et al. (2012) found that less adaptive emotional schemas were negatively related with psychological flexibility and more adaptive and flexible dimensions of emotional schemas were positively related to psychological flexibility. In the case of anxiety, interestingly the emotional schemas found to be most related with anxiety were those believed to be most related with experiential avoidance, namely the belief that emotions were out of control and would last indefinitely.

Determining these associations and given that the detrimental effects of early trauma is well known, determining the mediators in their association with emotional problems, will help clinicians determine the mechanisms and processes related with risk and maintenance of emotional distress. Psychological inflexibility is suggested as a transdiagnostic construct underlying mood disorders and so multiple constructs should be assessed in order to design effective interventions (Spinhoven et al., 2017). In light of research, it can be concluded that psychological flexibility is a protective factor in face of emotional distress (Trindade et al., 2020; Yasinski et al., 2020) and targeting its' enhancement will prove beneficial in the counseling process. In this case having determined emotional schemas as another factor affecting negative emotional state, the current results will help in broadening our understanding of the interrelated mechanisms.

Results of the present study further suggest that, how emotions are approached, assessed, and handled are interrelated with emotional distress. It is known that childhood 
experiences and learning set the grounds on which beliefs about emotions are shaped and further lead to avoidance strategies, and that these together evolve over time. Although as the amount of distress increases, so does the need to suppress and avoid, and individuals not experiencing distress would not have the need to avoid these private experiences (Batten et al., 2001), the amount of distress created by similar life events may differ between individuals due to differences in prior traumatic experiences, family environment, and multiple mechanisms affecting assessment of emotions. Also, similar feelings of distress will be evaluated differently. It may be that maltreated children, in their relationships with parents, were guided to believe that some emotions are bad and should be avoided and thus have learned to suppress and avoid, or even be afraid of them. Bowlby $(1973,1979)$ proposed that people build working models of the world and themselves in it, through which they perceive events, predict the future, and formulate plans. This model of self and the model of the attachment figure develop in an interactive reciprocally confirming way, which means that the child parent/caregiver relationship determines these models. As a result, the self-treatment of the child resembles the treatment from the parents and the child views himself/herself similar to the way the parent does. Such as, if the child is not accepted by the parent the representation of the self will develop as a person which is unacceptable. It is believed that these working models will apply to beliefs about emotions, which is an inseparable and integral part of the self, so if the self is not accepted neither are the emotions, and as a result the child believes that these emotions should be eliminated.

Relatedly, studies suggest the importance of family interactions, involving emotion socialization practices which affect the way the child learns to experience, regulate, and express emotions. Maternal emotion socialization approaches are linked to child adjustment (Cunningham et al., 2009) and unsupportive emotion socialization practices by parents may result in more negative views about sadness, which in turn mediates the relationship between these unsupportive parental attitudes and depression symptoms in adulthood (Boucher et al., 2013). Parents' lack of coaching of emotions and awareness of the child's emotions as well as their own, are related to internalized and externalized problems and anxiety disorders in children (Hurrell et al., 2017), and again parents' inhibition of display of negative emotions is in turn related to psychological distress in adulthood (Garside \& Klimes-Dougan, 2002). On the opposite side, coaching of emotions by parents is linked to fewer internalizing problems in adolescents (Stocker et al., 2007) which suggests the determining effect of the way parents model and approach their children's emotionality.

Another component in this case, can be related to the idea that it's not acceptable to experience negative emotions and that positive emotions are a sign of mental health, which is partly imposed by society. Hayes et al. (2006) state that trying to constantly avoid pain and feel good, is supported by the process in which negative emotions are evaluated, predicted, and avoided; this may lead to a conceptualization of the self, past, and future which controls behavior and enhances inflexibility, and results in detaching from values. This view emphasizes the fact that stressing values in the helping process would include preparing the individual for all kinds of emotions, and although it is not pleasant to feel hurtful emotions, trying to avoid them would lead to missed chances in life. Also, instead of categorizing emotions as negative and positive, and trying to eliminate painful emotions, the aim may be accepting that emotions can be painful and can cause suffering, and that this is part of human nature. The core of treatment is assessing the individuals' theory of emotion and helping in understanding that emotion regulation strategies involving trying to get rid of the emotion, negative evaluation of the emotion are the core of the problem, rather than the emotion per se (Leahy, 2015).

The fact that problematic emotion control strategies may be helpful for a while may confirm the belief that these emotions should be avoided, and may lead to further catastrophizing the problem, and the fact that these emotions keep coming back makes the individual feel more desperate in relying on these problematic strategies and further confirm beliefs about emotions (Leahy, 2015). On the other hand, if experiential avoidance is directed towards a meaningful, value riden activity instead of solely distracting oneself to avoid distressing thoughts, then it may not be that problematic and can even increase the sense of control over emotional discomfort (Kelso et al., 2020). Personal empowerment can be built by accepting painful emotions as part of life which may hold difficulties in itself (Leahy, 2015), but suffering is personal and unique for each individual. As a result, personal growth may arise with time merely as a choice, if one chooses so.

\section{Conclusion}

Psychological and behavioral inflexibility is related to the way individuals relate to what they think, and how they get stuck in this content, where thinking and what is thought about are treated as one, even though they are in fact dealing with thoughts and with the world as they cognitively organized it, rather than reality. Also starting from childhood individuals are expected to verbally explain and give reasons about their behavior, to justify what they are doing or feeling, and this goes hand in hand with the assumption that feelings and behaviors should be controlled, which in turn adds to the control these feelings have over our lives (Luoma et al., 2017). On the other hand, psychological flexibility is about experiencing life with its challenges, hardships, and opportunities instead of perceiving it as a problem which should be 
dealt with (Luoma et al., 2017), which is more than an all or none or constant state but differs from time to time.

Study findings helping to determine the emotional schemas most related with emotional distress, will help in identifying and working with these in psychotherapy and counseling. Accepting emotions or fearing about not being able to control them and believing that emotions will last once they get hold of the individual, strike out as several of the conceptualizations which can be investigated. In the case of anxiety disorders, it is known that the emotional schema of acceptance of emotions and use of acceptance based emotion regulation, may be beneficial in coping with anxiety about emotions (Leahy, 2002; Liverant et al., 2008). Another schema, fear of not being able to control emotions or emotional expressions is related to attributions about not being able to control feelings of anxiety and unpleasant events (Berg et al., 1998), and can be assessed and worked on to understand how it maintains and aggravates problematic ways of coping and how it intensifies distressing feelings (Leahy, 2015). A belief central to acceptance and control is the belief on the duration of emotions (Leahy, 2019), which is about believing that emotions are fixed, will last indefinitely, which is in turn found to determine anxiety and depression (Tirch et al., 2012). Beliefs about the duration of emotions may affect the way moderately depressed individuals perceive their emotional experiences and the way they regulate their emotions as a result of distress (Kneeland et al., 2020). Once certain upsetting emotions arise, maybe one of the major distressing points is the feeling that one will get stuck in these emotions, conversely, when emotions are believed to be malleable, then acceptance of feelings may increase (Ortner \& Pennekamp, 2020) which suggests that believing that emotions will last is an obstacle for acceptance of emotions, which is also another schema about emotions. Individuals may constantly try to avoid emotions in order to escape from the unpleasant results, which further enhances experiential avoidance and numbness in experiencing emotions, which are major obstacles in leading a meaningful and satisfying life and thus can be one of the targets in psychotherapy and counseling.

In conclusion, the present study highlights that how individuals approach, interpret and respond to their emotions are related to their level of distress. Certain stressful experiences may be easier or harder to handle for a person differing on the nature and intensity of the experience or event, and how important it is personally. Though the nature and intensity may change, if an emotion is accepted as negative, it is unpleasant. But for certain individuals any kind of unpleasant emotion can be extremely distressing, and still for some individuals these emotions may nearly always be extremely hard to handle. This study tries to pinpoint that one of the mechanisms behind these individual differences is childhood maltreatment. All children encounter differing levels of stressful events while growing up and sometimes some experiences may be considered traumatic. The interaction of traumatic experiences with genetic factors may even lead to self-harming behavior (Brodsky, 2016, as cited in De Berardis et al., 2018), and while some children who are exposed to traumatic life events may be relatively more resilient, others may generalize their fear, anger, unpleasant and hurtful experiences, and become highly sensitive and overreactive to certain stimuli (Cohen et al., 2017). They may suppress sensory information and intrusive cognitions related to their stressful or traumatic experiences (Goodyear-Brown, 2010), which may have longterm effects. Depression Patients with a history of childhood abuse were found to exhibit sensory sensitivity, avoidance and sensitivity towards sensory input, suggesting that as children, they may have acquired extreme sensory processing patterns (Serafini et al., 2016). These results indicate that many interrelated studies can be put together to get a wider picture of the mechanisms involved in coping with difficult emotions. That is to say, many interrelated mechanisms play a role in in how emotionally laden experiences are processed, and in this study childhood maltreatment is considered as one of these mechanisms. Relatedly, in face of stressful events, if children are not soothed, protected, supported, if parents don't provide necessary guidance in helping children understand what is happening, talk with them about their feelings and thoughts, they may be overwhelmed and will fail to give meaning to their bodily sensations, strong feelings, and what's going on in their mind. Putting it all together, emotional schemas and psychological inflexibility help us understand and work with individuals who have grown up with such experiences and relatedly have adopted problematic coping strategies, help them genuinely experience their feelings without being threatened, and help them lead a more genuine, meaningful, and fulfilling life.

Also, the fact that findings from this Turkish sample are in line with previous research conducted in other cultures, suggests a multi-cultural aspect of these factors and their relationship with each other.

\section{Limitations and Future Study Directions}

The study was cross-sectional, which doesn't allow for causal inferences. Due to the COVID-19 restrictions it was not possible to collect data from a hospital or clinical setting and the sample could only be reached online and thus a cross-sectional design was implemented. Possible childhood trauma including complex trauma wasn't assessed, and the presence of other adverse childhood experiences which may have led to emotional difficulties is not known. Relatedly participants may have been to psychotherapy for related issues. Also the possible effects of the COVID pandemic on the mental health of participants it is not known. 
Similar studies with individuals diagnosed with depression and anxiety will help generalize findings. Qualitative studies including in-depth interviews conducted with clinical samples will help in our understanding of the factors related with childhood maltreatment which creates risk for mental health problems. Also assessing anxiety sensitivity, factors adding to the maintenance and relapse of depression and anxiety disorders as well as protective factors, will help broaden our understanding of the interrelations.

\section{Declarations}

Conflict of Interest The author declares no conflict of interest.

\section{References}

Akbari, M., \& Khanipour, H. (2018). The transdiagnostic model of worry: The mediating role of experiential avoidance. Personality and Individual Differences, 135, 166-172. https://doi.org/10. 1016/j.paid.2018.07.011

Bardeen, J. R., Fergus, T. A., \& Orcutt, H. K. (2014). The moderating role of experiential avoidance in the prospective relationship between anxiety sensitivity and anxiety. Cognitive Therapy Research, 38, 465-471. https://doi.org/10.1007/ s10608-014-9614-z

Batmaz, S., Kaymak, S. U., Kocbiyik, S., \& Turkcapar, M. H. (2014). Metacognitions and emotional schemas: A new cognitive perspective for the distinction between unipolar and bipolar depression. Comprehensive Psychiatry, 55(7), 1546-1555. https://doi.org/10. 1016/j.comppsych.2014.05.016

Batmaz, S., \& Özdel, K. (2015). Psychometric properties of the Turkish version of the Leahy emotional schema Scale-II. Anadolu Psikiyatri Dergisi (Journal of Anadolu Psychiatry), 16(Özel say1 - special issue1), 23-30. https://doi.org/10.5455/ apd. 170597

Batten, S. V., Follette, V. M., \& Aban, I. B. (2001). Experiential avoidance and high-risk sexual behavior in survivors of child sexual abuse. Journal of Child Sexual Abuse, 10(2), 101-20. https://doi. org/10.1300/J070v10n02_06

Baugh, L. M., Cox, D. W., Young, R. A., \& Kealy, D. (2019). Partner trust and childhood emotional maltreatment: The mediating and moderating roles of maladaptive schemas and psychological flexibility. Journal of Contextual Behavioral Science, 12, 66-73. https://doi.org/10.1016/j.jcbs.2019.02.001

Berg, C. Z., Shapiro, N., Chambless, D. L., \& Ahrens, A. H. (1998). Are emotions frightening? II: An analogue study of fear of emotion, interpersonal conflict, and panic onset. Behavior Research and Therapy, 36(1), 3-15. https://doi.org/10.1016/S00057967(97)10027-4

Berking, M., Poppe, C., Luhmann, M., Wupperman, P., Jaggi, V., \& Seifritz, E. (2012). Is the association between various emotionregulation skills and mental health mediated by the ability to modify emotions? Results from two cross-sectional studies. Journal of Behavior Therapy \& Experimental Psychiatry, 43, 931-937. https://doi.org/10.1016/j.jbtep.2011.09.009

Bernstein, D. P., Fink, L., Handelsman, L., Foote, J., Lovejoy, M., Wenzel, K., Sapareto, E., \& Ruggiero, J. (1994). Initial reliability and validity of a new retrospective measure of child abuse and neglect. American Journal of Psychiatry, 151(8), 1132-1136. https://doi.org/10.1176/ajp.151.8.1132
Biglan, A., Gau, J. M., Jones, L. B., Hinds, E., Rusby, J. C., Cody, C., \& Sprague, J. (2015). The role of experiential avoidance in the relationship between family conflict and depression among early adolescents. Journal of Contextual Behavioral Science, 4, 30-36. https://doi.org/10.1016/j.jcbs.2014.12.001

Bond, F. W., Hayes, S. C., Baer, R. A., Carpenter, K. M., Guenole, N., Orcutt, H. K., Waltz, T., \& Zettle, R. D. (2011). Preliminary psychometric properties of the acceptance and action questionnaire-II: A revised measure of psychological inflexibility and experiential avoidance. Behavior Therapy, 42(4), 676-688. https://doi.org/10.1016/j.beth.2011.03.007

Boucher, M. E., Lecours, S., Philippe, F. L., \& Arseneault, S. (2013). Parental socialization of emotion and depression in adulthood: The role of attitudes toward sadness. European Review of Applied Psychology, 63(1), 15-23. https://doi.org/10.1016/j. erap.2012.11.003

Boykin, D. M., Himmerich, S. J., Pinciotti, C. M., Miller, L. M., Miron, L. R., \& Orcutt, H. K. (2018). Barriers to self-compassion for female survivors of childhood maltreatment: The roles of fear of self-compassion and psychological inflexibility. Child Abuse \& Neglect, 76, 216-224. https://doi.org/10.1016/j. chiabu.2017.11.003

Bowlby, J. (1973). Attachment and loss: Separation, anxiety and anger (Vol. 2). Basic Books.

Bowlby, J. (1979). The making and breaking of affectional bonds. Tavistock.

Brodsky, B. S. (2016). Early Childhood Environment and Genetic Interactions: The Diathesis for Suicidal Behavior. Current Psychiatry Reports, 18, 86.

Caldwell, J. G., Krug, M. K., Carter, C. S., \& Minzenberg, M. J. (2014). Cognitive control in the face of fear: Reduced cognitiveemotional flexibility in women with a history of child abuse. Journal of Aggression, Maltreatment \& Trauma, 23(5), 454472. https://doi.org/10.1080/10926771.2014.904466

Coates, A. A., \& Messman-Moore, T. L. (2014). A structural model of mechanisms predicting depressive symptoms in women following childhood psychological maltreatment. Child Abuse \& Neglect, 38(1), 103-113. https://doi.org/10.1016/j.chiabu.2013.10.005

Cohen, J. (1988). Statistical power analysis for the behavioral sciences. Lawrence Erlbaum.

Cohen, J. A., Mannarino, A. P., \& Deblinger, E. (2017). Treating trauma and traumatic grief in children and adolescents. The Guilford Press.

Cohen, J. R., \& Thakur, H. (2021). Developmental consequences of emotional abuse and neglect in vulnerable adolescents: A multiinformant, multi-wave study. Child Abuse \& Neglect, 111, 104811. https://doi.org/10.1016/j.chiabu.2020.104811

Cunningham, J. N., Kliewer, W., \& Garner, P. W. (2009). Emotion socialization, child emotion understanding and regulation, and adjustment in urban African American families: Differential associations across child gender. Developmental Psychopathology, 21(1), 261-283. https://doi.org/10.1017/S0954579409000157

De Berardis, D., Fornaro, M., Valchera, A., Cavuto, M., Perna, G., Di Nicola, M., et al. (2018). Eradicating suicide at its roots: Preclinical bases and clinical evidence of the efficacy of ketamine in the treatment of suicidal behaviors. International Journal of Molecular Sciences, 19(10), 2888. https://doi.org/10.3390/ ijms19102888

Li, E. T., Carracher, L., \& Bird, T. (2020a). Linking childhood emotional abuse and adult depressive symptoms: The role of mentalizing incapacity. Child Abuse \& Neglect, 99, 104253. https:// doi.org/10.1016/j.chiabu.2019.104253

Edalati, H., Krank, M. D., \& Schütz, C. G. (2020). Childhood maltreatment and perceived stress in individuals with concurrent psychiatric disorders. Journal of Aggression, Maltreatment \& Trauma, 29(1), 22-37. https://doi.org/10.1080/10926771.2019.1595802 
Edwards, E. R., Liu, Y., Ruiz, D., Brosowsky, N. P., \& Wupperman, P. (2020). Maladaptive emotional schemas and emotional functioning: Evaluation of an integrated model across two independent samples. Journal of Rational-Emotive \& Cognitive-Behavior Therapy. https://doi.org/10.1007/ s10942-020-00379-8

Fischer, T. D., Smout, M. F., \& Delfabbro, P. H. (2016). The relationship between psychological flexibility, early maladaptive schemas, perceived parenting and psychopathology. Journal of Contextual Behavioral Science, 5(3), 169-177. https://doi.org/10.1016/j.jcbs. 2016.06.002

Fonseca, S., Trindade, I. A., Mendes, A. L., \& Ferreira, C. (2020). The buffer role of psychological flexibility against the impact of major life events on depression symptoms. Clinical Psychologist, 24(1), 82-90. https://doi.org/10.1111/cp.12194

Garside, R. B., \& Klimes-Dougan, B. (2002). Socialization of discrete negative emotions: Gender differences and links with psychological distress. Sex Roles, 47, 115-128. https://doi.org/10.1023/A: 1021090904785

Ghazanfari, F., Rezaei, M., \& Rezaei, F. (2018). The mediating role of repetitive negative thinking and experiential avoidance on the relationship between childhood trauma and depression. Archives of Psychiatric Nursing, 32, 432-438. https://doi.org/10.1016/j. apnu.2017.12.010

Goodman, M. L., Gutarra, C., Billingsley, K. M., Keiser, P. H., \& Gitari, S. (2017). Childhood exposure to emotional abuse and later life stress among Kenyan women: A mediation analysis of cross-sectional data. Anxiety, Stress \& Coping, 30(4), 469-483. https://doi.org/10.1080/10615806.2016.1271876

Goodyear-Brown, P. (2010). Play therapy with traumatized children: a prescriptive approach. Wiley.

Hayes, S. C., Wilson, K. G., Gifford, E. V., Follette, V. M., \& Strosahl, K. D. (1996). Experiential avoidance and behavioral disorders: A functional dimensional approach to diagnosis and treatment. Journal of Consulting and Clinical Psychology, 64(6), 1152-1168. https://doi.org/10.1037/0022-006X.64.6.1152

Hayes, S. C., \& Smith, S. (2005). Get out of your mind and into your life : The new acceptance and commitment therapy. New Harbinger Publications Inc.

Hayes, S. C., Luoma, J. B., Bond, F. W., Masuda, A., \& Lillis, J. (2006). Acceptance and commitment therapy: Model, processes and outcomes. Behavior Research and Therapy, 44(1), 1-25. https://doi. org/10.1016/j.brat.2005.06.006

Hayes, S. C., Strosahl, K. D., \& Wilson, K. G. (2012). Acceptance and commitment therapy: The process and practice of mindful change. The Guilford Press.

Hurrell, K. E., Houwing, F. L., \& Hudson, J. L. (2017). Parental meta-emotion philosophy and emotion coaching in families of children and adolescents with an anxiety disorder. Journal of Abnormal Child Psychology, 45, 569-582. https://doi.org/10. 1007/s10802-016-0180-6

Karekla, M., \& Panayiotou, G. (2011). Coping and experiential avoidance: Unique or overlapping constructs? Journal of Behavior Therapy and Experimental Psychiatry, 42(2), 163-170. https:// doi.org/10.1016/j.jbtep.2010.10.002

Kashdan, T., \& Rottenberg, J. (2010). Psychological flexibility as a fundamental aspect of health. Clinical Psychology Review, 30(7), 865-878. https://doi.org/10.1016/j.cpr.2010.03.001

Kelso, K. C., Kashdan, T. B., Imamoğlu, A., \& Ashraf, A. (2020). Meaning in life buffers the impact of experiential avoidance on anxiety. Journal of Contextual Behavioral Science, 16, 192-198. https://doi.org/10.1016/j.jcbs.2020.04.009

Kneeland, E. T., Goodman, F. R., \& Dovidio, J. F. (2020). Emotion beliefs, emotion regulation, and emotional experiences in daily life. Behavior Therapy, 51(5), 728-738. https://doi.org/10.1016/j. beth.2019.10.007
Krause, E. D., Mendelson, T., \& Lynch, T. R. (2003). Childhood emotional invalidation and adult psychological distress: The mediating role of emotional inhibition. Child Abuse \& Neglect, 27, 199-213.

Leahy, R. L. (2002). A model of emotional schemas. Cognitive and Behavioral Practice, 9(3), 177-190. https://doi.org/10.1016/ S1077-7229(02)80048-7

Leahy, R. L. (2015). Emotional schema therapy. The Guilford Press.

Leahy, R. L. (2018). Introduction: Emotional schemas and emotional schema therapy. International Journal of Cognitive Therapy, 12, 1-4. https://doi.org/10.1007/s41811-018-0038-5

Leahy, R. L. (2019). Emotional schema therapy: Distinctive features. Routledge.

Li, E. T., Carracher, E., \& ve Bird, T. (2020b). Linking childhood emotional abuse and adult depressive symptoms: The role of mentalizing incapacity. Child Abuse \& Neglect, 99, 104253. https://doi. org/10.1016/j.chiabu.2019.104253

Li, Q., Guo, L., Zhang, S., Wang, W., Li, W., Chen, X., Shi, J., Lu, C., $\&$ ve McIntyre, R. S. (2021). The relationship between childhood emotional abuse and depressive symptoms among Chinese college students: The multiple mediating effects of emotional and behavioral problems. Journal of Affective Disorders, 288, 129-135. https://doi.org/10.1016/j.jad.2021.03.074

Liu, S., Zhou, N., Dong, S., Wang, Z., \& veHao, Y. (2019). Maternal childhood emotional abuse predicts Chinese infant behavior problems: Examining mediating and moderating processes. Child Abuse \& Neglect, 88, 307-316. https://doi.org/10.1016/j.chiabu. 2018.12.006

Lovibond, P. F., \& Lovibond, S. H. (1995). The structure of negative emotional states: Comparison of the Depression Anxiety Stress Scales (DASS) with the Beck depression and anxiety inventories. Behaviour Research and Therapy, 33(3), 335-343. https://doi.org/ 10.1016/0005-7967(94)00075-U

Luoma, J. B., Hayes, S. C., \& Walser, R. D. (2017). Learning ACT: An acceptance \& commitment therapy skills training manual for therapists. New Harbinger Publications Inc.

Makriyianis, H. M., Adams, E. A., Lozano, L. L., Mooney, T. A., Morton, C., \& Liss, M. (2019). Psychological inflexibility mediates the relationship between adverse childhood experiences and mental health outcomes. Journal of Contextual Behavioral Science, 14, 82-89. https://doi.org/10.1016/j.jcbs.2019.09.007

Mazloom, M., Yaghubi, H., \& Mohammadkhani, S. (2016). Posttraumatic stress symptom, metacognition, emotional schema and emotion regulation: A structural equation model. Personality and Individual Differences, 88, 94-98. https://doi.org/10.1016/j.paid. 2015.08.053

McKay, M., Lev, A., \& Skeen, M. (2012). Acceptance and commitment therapy for interpersonal problems: Using mindfulness, acceptance, and schema awareness to change interpersonal behaviors. New Harbinger Publications Inc.

Moraes, I. L., Sampaio, P. F., Reichenheim, M. E., \& ve da veiga, G. V. (2018). The intertwined effect of lack of emotional warmth and child abuse and neglect on common mental disorders in adolescence. Child Abuse \& Neglect, 83, 74-82. https://doi.org/10. 1016/j.chiabu.2018.07.008

Moroz, M., \& Dunkley, D. M. (2019). Self-critical perfectionism, experiential avoidance, and depressive and anxious symptoms over two years: A three-wave longitudinal study. Behaviour Research and Therapy, 112, 18-27. https://doi.org/10.1016/j.brat.2018.11.006

Pearce, J. W., \& vePezzot-Pearce, T. D. (2007). Psychotherapy of abused and neglected children. The Guilford Press.

Predatu, R., David, D. O., \& Maffei, A. (2020). The effects of irrational, rational, and acceptance beliefs about emotions on the emotional response and perceived control of emotions. Personality and Individual Differences, 155, 109712. https://doi.org/10. 1016/j.paid.2019.109712 
Ortner, N. M., \& Pennekamp, P. (2020). Emotion malleability beliefs and event intensity and importance predict emotion regulation in daily life. Personality and Individual Differences, 159, 109887. https://doi.org/10.1016/j.paid.2020.109887

Ramaghani, N. A. H., Rezaei, F., Sepahvandi, M. A., Gholamrezaei, S., \& Mirderikvand, F. (2019). The mediating role of the metacognition, time perspectives and experiential avoidance on the relationship between childhood trauma and post-traumatic stress disorder symptoms. European Journal of Psychotraumatology, 10(1), 1648173. https://doi.org/10.1080/20008198.2019.1648173

Reddy, M. K., Pickett, S. M., \& Orcutt, H. K. (2006). Experiential avoidance as a mediator in the relationship between childhood psychological abuse and current mental health symptoms in college students. Journal of Emotional Abuse, 6(1), 67-85. https:// doi.org/10.1300/J135v06n01 04

Reffi, A. N., Boykin, D. M., \& Orcutt, H. K. (2019). Examining pathways of childhood maltreatment and emotional dysregulation using self-compassion. Journal of Aggression, Maltreatment \& Trauma, 28(10), 1269-1285. https://doi.org/10.1080/10926771. 2018.1485810

Rezaei, R., Ghazanfari, F., \& Fatemeh, R. (2016). The role of childhood trauma, early maladaptive schemas, emotional schemas and experimental avoidance on depression: A structural equation modeling. Psychiatry Research, 246, 407-414. https://doi.org/10. 1016/j.psychres.2016.10.037

Richardson, C. M. E., \& Jost, S. A. (2019). Psychological flexibility as a mediator of the association between early life trauma and psychological symptoms. Personality and Individual Differences, 141, 101-106. https://doi.org/10.1016/j.paid.2018.12.029

Roche, A. I., Kroska, E. B., Miller, M. L., Kroska, S. K., \& O'Hara, M. W. (2019). Childhood trauma and problem behavior: Examining the mediating roles of experiential avoidance and mindfulness processes. Journal of American College Health, 67(1), 17-26. https://doi.org/10.1080/07448481.2018.1455689

Rosenthal, M. Z., Rasmussen-Hall, M. L., \& Palm, K. M. (2005). Chronic avoidance helps explain the relationship between severity of childhood sexual abuse and psychological distress in adulthood. Journal of Child Sexual Abuse, 14, 25-41. https://doi.org/ 10.1300/J070v14n04 02

Serafini, G., Gonda, X., Pompili, M., Rihmer, Z., Amore, M., \& EngelYeger, B. (2016). The relationship between sensory processing patterns, alexithymia, traumatic childhood experiences, and quality of life among patients with unipolar and bipolar disorders. Child Abuse \& Neglect, 62, 39-50.

Sheffler, J. L., Piazza, J. R., Quinn, J. M., Sachs-Ericsson, N. J., \& Stanley, I. H. (2019). Adverse childhood experiences and coping strategies: Identifying pathways to resiliency in adulthood. Anxiety, Stress \& Coping, 32(5), 594-609. https://doi.org/10.1080/ 10615806.2019.1638699

Silberstein, L. R., Tirch, D., \& Leahy, R. L. (2012). Mindfulness, psychological flexibility and emotional schemas. International Journal of Cognitive Therapy, 5(4), 406-419. https://doi.org/10. 1521/ijct.2012.5.4.406

Spinhoven, P., Elzinga, B. M., Van Hemert, A. M., de Rooij, M., \& Penninx, B. W. (2016). Childhood maltreatment, maladaptive personality types and level and course of psychological distress: A six-year longitudinal study. Journal of Affective Disorders, 191, 100-108. https://doi.org/10.1016/j.jad.2015.11.036

Spinhoven, P., van Hemert, A. M., \& Penninx, B. W. J. H. (2017). Experiential avoidance and bordering psychological constructs as predictors of the onset, relapse and maintenance of anxiety disorders: One or many? Cognitive Therapy Research, 41(6), 867-880. https://doi.org/10.1007/s10608-017-9856-7

Stocker, C. M., Richmond, M. K., Rhoades, G. K., \& Kiang, L. (2007). Family emotional processes and adolescents' adjustment. Social
Development, 16, 310-325. https://doi.org/10.1111/j.1467-9507. 2007.00386.x

Şar, V., Öztürk, E., \& İkikardeş, E. (2012). Validity and reliability of the Turkish Version of the Childhood Trauma Questionnaire (CTQ). Turkiye Klinikleri Tip Bilimleri Dergisi (turkiye Klinikleri Journal of Medical Sciences), 32(4), 1054-1063. https://doi.org/ 10.5336/medsci.2011-26947

Şar, V., Necef, I., Mutluer, T., Fatih, P., \& Türk-Kurtça, T. (2021). A revised and expanded version of the Turkish Childhood Trauma Questionnaire (CTQ-33): Overprotection- overcontrol as additional factor. Journal of Trauma and Dissociation, 22(1), 35-51. https://doi.org/10.1080/15299732.2020.1760171

Tabachnick, B. G., \& Fidell, L. S. (2001). Using Multivariate statistics. Allyn and Bacon.

Tirch, D., Leahy, R. L., Silberstein, L. R., \& Melwani, P. S. (2012). Emotional schemas, psychological flexibility, and anxiety: The role of flexible response patterns to anxious arousal. International Journal of Cognitive Therapy, 5(4), 380-391. https://doi.org/10. 1521/ijct.2012.5.4.380

Trindade, I. A., Mendes, A. L., \& Ferreira, N. B. (2020). The moderating effect of psychological flexibility on the link between learned helplessness and depression symptomatology: A preliminary study. Journal of Contextual Behavioral Science, 15, 68-72. https://doi.org/10.1016/j.jcbs.2019.12.001

Tull, M. T., \& Gratz, K. L. (2008). Further examination of the relationship between anxiety sensitivity and depression: The mediating role of experiential avoidance and difficulties engaging in goaldirected behavior when distressed. Journal of Anxiety Disorders, 22(2), 199-210. https://doi.org/10.1016/j.janxdis.2007.03.005

Veilleux, J. C., Pollert, G. A., Skinner, K. D., Chamberlain, K. D., Baker, D. E., \& Hill, M. A. (2021). Individual beliefs about emotion and perceptions of belief stability are associated with symptoms of psychopathology and emotional processes. Personality and Individual Differences, 171, 110541. https://doi.org/10. 1016/j.paid.2020.110541

Veilleux, J. C., Chamberlain, K. D., Baker, D. E., \& Warner, E. A. (2020). Disentangling beliefs about emotions from emotion schemas. Journal of Clinical Psychology, 77(4), 1068-1089. https:// doi.org/10.1002/jclp.23098

Yasinski, C., Hayes, A. M., Ready, C. B., Abel, A., Görg, N., \& Kuyken, W. (2020). Processes of change in cognitive behavioral therapy for treatment-resistant depression: Psychological flexibility, rumination, avoidance, and emotional processing. Psychotherapy Research, 30(8), 983-997. https://doi.org/10.1080/ 10503307.2019.1699972

Yavuz, F., Ulusoy, S., Iskin, M., Esen, F. B., Burhan, H. S., Karadere, M. E., \& Yavuz, N. (2016). Turkish version of Acceptance and Action Questionnaire-II (AAQ-II): A reliability and validity analysis in clinical and non-clinical samples. Klinik Psikofarmakoloji Bulteni - Bulletin of Clinical Psychopharmacology, 26(4), 397-408. https://doi.org/10.5455/bcp.20160223124107

Yildirim, A., Boysan, M., \& Kefeli, M. C. (2018). Psychometric properties of the Turkish version of the Depression Anxiety Stress Scale-21 (DASS-21). British Journal of Guidance \& Counselling, 46(5), 582-595. https://doi.org/10.1080/03069885.2018.1442558

Liverant, G. I., Brown, T. A., Barlow, D. H., \& Roemer, L. (2018). Emotion regulation in unipolar depression: the effects of acceptance and suppression of subjective emotional experience on the intensity and duration of sadness and negative affect. Behavior Research and Therapy, 46(11), 1201-1209. https://doi.org/10. 1016/j.brat.2008.08.001.

Publisher's Note Springer Nature remains neutral with regard to jurisdictional claims in published maps and institutional affiliations. 